\title{
Reviewer Acknowledgements for Global Journal of Health Science, Vol. 8 , No. 5
}

Global Journal of Health Science wishes to acknowledge the following individuals for their assistance with peer review of manuscripts for this issue. Their help and contributions in maintaining the quality of the journal is greatly appreciated.

Global Journal of Health Science is recruiting reviewers for the journal. If you are interested in becoming a reviewer, we welcome you to join us. Please find the application form and details at http://www.ccsenet.org/reviewer and e-mail the completed application form to gjhs@ccsenet.org.

\section{Reviewers for Volume 8, Number 5}

Alexander Domnich

Ambika Eranki

Asad Ali Khan Afridi

Bashiru I. I. Saeed

Chelsea Booth

Dalia Salah El-Deen El-Sedawy

Darampal Dambhare

Dilek Pirim

Dongxu Fu

E. Pepelassi

Esteban Ortiz-Prado

Evangelia Mavrikaki

Evanthia Sakellari

Gavric Zivana

Giovana Fernandes

Hadii Mohammed Mamudu

Joanne Yeoh

Johnson Jennifer

Jose R. Cordon

Kartheek Balapala

Leila Hamzehgardeshi
Liu Liu

Loray Daws

Marcela Quiñones

Maria Malliarou

Matteo Vitali

Meng Zhao

Montarat Thavorncharoensap

Nima Hafezi Nejad

Nishith Vayada

Pavlos Sarafis

Pelechas Eleftherios

Radu-Iulian Spataru

Raywat Deonandan

Satoshi Horiuchi

Shaillay Dogra

Steven Hoffman

Torill Larsen

Tracie L. Pasold

Tsan Yang

Yasam Kemal Akpak

Yevgeniy Samyshkin 